\title{
NEW EMBEDDING FORMULATIONS USING QUETOL 651
}

\author{
E. Ann Ellis
}

Microscopy and Imaging Center, Texas A \& M University, College Station, TX 77843

Quetol 651, ethylene glycol diglycidal ether (glycerol polyglycidal ether), was first introduced [1] as a component of low and ultra low viscosity [2] epoxy embedding media. Quetol 651 is a light colored resin with a viscosity of $15 \mathrm{cps}$ at $25^{\circ} \mathrm{C}$ and serves to maintain a reduced viscosity and longer pot life in resin mixtures. Quetol 651 was used in two different formulations with the higher viscosity epoxy resins, LX-112 and Araldite 502, to produce lower viscosity formulations with longer pot life, good cutting properties, good contrast and stability in the electron beam.

Formulations of the aliphatic Epon 812 replacement resin, LX-112, with Quetol 651 and the anhydride, dodecenyl succinic anhydride (DDSA), were made with an anhydride:epoxide ratio of 1.0; benzyl dimethylamine (BDMA) was the accelerator (Formulation 1). [Eponate 12 can be substituted for LX-112 and results in a comparable embedding medium with similar reduction in viscosity, good cutting properties and good beam stability.] Quetol 651 was substituted for Epon 812 in Mollenhauer's [3] formulation of Epon 812 and Araldite 502 with DDSA, BDMA and an anhydride:epoxide ration of 0.8 (Formulation 2). All formulations were done by gravimetric (weight) methods and BDMA was used at the rate of $0.18 \mathrm{ml} / 10$ grams of stock embedding mixture. In order to insure consistent embedding mixtures the following protocol was used: 1) in a clean, dry container with a tight fitting lid, DDSA and Quetol 651 were mixed using a magnetic stirrer; 2) then LX-112 or Araldite 502 was added and stirred again until there were no visible mixing striations. Finally, the low viscosity accelerator, BDMA, was added and the mixture was stirred until thoroughly mixed. Viscosities of the embedding mixtures were determined with a Brookfield Microviscometer. Formulations of these mixtures, calculated on the basis of 10 gram aliquots, are given in Table 1. Table 2 shows the method for calculating or adjusting the ratios of Quetol 651 and higher viscosity epoxy resins to make new formulations with known, anhydride:epoxide ratios and consistent cutting properties. Viscosities of these new formulations, as compared to other standard resin formulations (Table 3), were reduced by $25-50 \%$.

Appropriate sized batches of embedding medium should be made immediately before use; unused resin can be kept for the day in a closed, sealed container in a desiccator. All components of the embedding formulation should be handled in a fume hood using disposable pipettes (or syringes), beakers and gloves. Waste embedding media should be polymerized before disposal. Although Quetol 651 is miscible with water, ethanol, acetone and other dehydrating agents and can be used as a dehydrating agent, extreme care should be taken to avoid absorbing atmospheric moisture in climates with high humidity since this will inhibit proper polymerization of specimen blocks. The formulations presented here have been used extensively by the author since 1984 with difficult specimens of optic nerve and other ocular tissues for studies involving cytochemical localizations and colloidal gold labeled immunocytochemistry [4].

References

[1] H. Kushida, J Electron Microsc, 23(1974)197. 
[2] D. L. Ringo et al., Proc Electron Microscopy Soc Amer, 37(1979)348.

[3] H. H. Mollenhauer, Stain Technol, 39(1964)111.

[4] E. A. Ellis et al., Free Rad Biol Med, 24(1998)120.

[5] A. R. Spurr, J Ultrastruct Res, 26(1969)31.

TABLE 1. New Embedding Formulations Using Quetol 651*

\begin{tabular}{lclc}
\hline LX-112 & & ARALDITE 502 \\
FORMULATION 1 & & FORMULATION 2 \\
& & & \\
\hline DDSA & $6.98 \mathrm{~g}$ & DDSA & $5.66 \mathrm{~g}$ \\
QUETOL 651 & $1.14 \mathrm{~g}$ & QUETOL 651 & $1.16 \mathrm{~g}$ \\
LX-112 & $1.88 \mathrm{~g}$ & ARALDITE 502 & $3.18 \mathrm{~g}$ \\
BDMA & $0.18 \mathrm{ml}$ & BDMA & $0.18 \mathrm{ml}$ \\
\hline
\end{tabular}

* Source for Quetol 651: Most electron microscopy supply companies in the United States sell this product in a kit or as a separate item.

TABLE 2. Formulation Calculation

\begin{tabular}{|c|c|c|c|}
\hline $\begin{array}{l}\text { COMPONENT } \\
{\text { Mol. Wt. }{ }^{A} \text { or WPE }}^{\text {B }}\end{array}$ & $\begin{array}{c}\text { DDSA } \\
266^{\mathrm{A}}\end{array}$ & $\begin{array}{c}\text { QUETOL } 651 \\
87^{\mathrm{B}}\end{array}$ & $\begin{array}{c}\mathrm{LX}-112 \\
143^{\mathrm{B}}\end{array}$ \\
\hline Molar Ratios & 1.0 & $0.5^{* *}$ & $0.5^{* *}$ \\
\hline Proportion by Wt. & $(266 \times 1.0)=266$ & $(87 \times 0.5)=43.5$ & $(143 \times 0.5)=71.5$ \\
\hline Total Wt. & $266+43.5+71.5=381$ & & \\
\hline \% by Wt. & $266 / 381=69.8 \%$ & $43.5 / 381=11.4 \%$ & $71.5 / 381=18.8 \%$ \\
\hline Formulation for $10 \mathrm{~g}$ & $10 \mathrm{~g} \times 0.698=6.98 \mathrm{~g}$ & $10 \mathrm{~g} \times 0.114=1.14 \mathrm{~g}$ & $10 \mathrm{~g} \times 0.188=1.88 \mathrm{~g}$ \\
\hline
\end{tabular}

${ }^{* *}$ The sum of the molar ratios for the epoxy components should equal 1.0.

TABLE 3. Viscosities of Standard Embedding Formulations and Formulations Using Quetol 651

\begin{tabular}{lc}
\hline EMBEDDING FORMULATION & VISCOSITY [CENTPOISE] AT $25^{\circ} \mathrm{C}$ \\
\hline EPON 812/ARALDITE 502 & 2500 \\
LUFT'S EPON 812 & 550 \\
LUFT'S LX-112 & 340 \\
ARALDITE 502 and DDSA & 1075 \\
LX-112 and DDSA & 380 \\
ARALDITE 502/QUETOL 651 & 800 \\
LX-112/QUETOL 651 & 250 \\
SPURR LOW VISCOSITY & 60 \\
\hline
\end{tabular}

